\title{
STK11 Gene Mutation is a Negative Prognostic Predictor for Metastatic Cancer Patients Treated with Immune Checkpoint Inhibitors
}

\author{
Yongping $\mathrm{Liu}^{1,2,4^{*}}$, lin $\mathrm{Chen}^{3,4}$, ShenLi Zhang ${ }^{3,4}$, Qiufeng $\mathrm{Qi}^{1,4}$, Ming Zhu ${ }^{1,4}$, Yun Peng ${ }^{1,4}$, Yang Ling ${ }^{1,2}$ \\ ${ }^{1}$ Clinical Oncology Laboratory, Changzhou Tumor Hospital Affiliated to Soochow University, Changzhou, China \\ ${ }^{2}$ Department of Oncology, Changzhou Tumor Hospital Affiliated to Soochow University, Changzhou, China \\ ${ }^{3}$ The Third Affiliated Hospital of Soochow University, Changzhou, China \\ ${ }^{4}$ Central Laboratory, Changzhou Tumor Hospital Affiliated to Soochow University, Changzhou, China
}

\section{Abstract}

Immune checkpoint inhibitors open a new door for tumor immunotherapy, but it should not be ignored that some tumor patients are still insensitive to this kind of therapy. The purpose of this study is to analyze the prognostic value of STK11 mutations in metastatic cancer patients treated with ICIs. Information regarding somatic gene mutations of STK11 and patients' survival time in advanced cancer patients receiving ICIs treatment was downloaded from the cbioportal database. The prognostic value of STK11 gene mutation was analyzed by Log-rank test and multivariate Cox proportional hazards regression analysis. The somatic mutation frequencies of STK11 among all patients were $6.14 \%$, and STK11 gene mutations were mainly distributed in patients with non-small cell lung cancer. The results showed STK11 gene mutation status was associated with shorter survival time, the hazard ratio is $1.746(P<0.0001)$. The somatic mutations of STK11 were also associated with shorter overall survival in NSCLC patients $(P=0.017)$. Multivariate Cox proportional hazards regression analysis showed $S T K 11$ gene mutation was significantly associated with overall survival $(\mathrm{HR}=1.567,95 \% \mathrm{CI}$ : 1.209-2.029, $P=0.001$ ). The results of this study suggested that $S T K 11$ gene mutation could be used as a negative prognostic marker in metastatic cancer patients receiving ICIs treatment.

Keywords: STK11; gene mutation; cancer, prognosis; Immune checkpoint inhibitor.

Copyright @ 2019: This is an open-access article distributed under the terms of the Creative Commons Attribution license which permits unrestricted use, distribution, and reproduction in any medium for non-commercial use (NonCommercial, or CC-BY-NC) provided the original author and source are credited.

\section{INTRODUCTION}

Tumor mutational burden is an emerging biomarker to predict therapeutic response in advanced cancer patients receiving immune checkpoint inhibitor (ICI) therapies, including antibodies that target CTLA-4 or PD-1/PD-L1[1-4]. MSK-IMPACT panel and some other panels, based on targeted sequencing technology, have been approved by FDA to evaluate TMB. The theoretical basis of TMB as a predictive marker of ICI therapy is that the higher the TMB, the more new antigens are produced, which could trigger anti-tumor immunity $[5,6]$.

The inactivated STK11 gene mutation is one of the most common tumor-suppressor gene mutations, especially in non-small cell lung cancer. Recent studies have shown that STK11/ LKB1 inactivated mutation may be a negative predictor of immunocheckpoint inhibitor therapy [7, 8]. Shohei Koyama reported that tumors with $S T K 11 / L K B 1$ inactivating mutations were associated with reduced expression of PD -L1 and the decreased number of tumor-infiltrating lymphocytes, which may lead to ineffectiveness of immunocheckpoint inhibitor therapy[9].

To further explore the predictive value of STK11 mutation in ICIs therapy, we downloaded 1661 tumor patients' information from cBioPortal data base, including STK11 mutation status, patient survival time, ICI type, tumor type, TMB status and other clinicopathological characteristics. We analyzed the prognostic value of somatic gene mutation of STK11 in 1661 advanced cancer patients treated with ICIs, as well as the associations between somatic mutation of STK11 gene and TMB and other genes.

\section{MATERIALS AND METHODS Gene databases}

Somatic mutation of STK11 gene and patients' survival time in advanced cancer patients treated with ICIs was downloaded from the cBioPortal database, an open access database that is publicly available at http://www.cbioportal.org [10, 11].We chose MSKIMPACT Clinical Sequencing Cohort (MSKCC, Nat 
Genet 2019)as our data source, which contained only advanced cancer patients treated with ICIs[2]. The total number of samples was 1661. Only data including mutations was obtained. The queried genes contain one gene: STK11 gene. Information regarding STK11 gene mutation and survival data from 1661 patients among various cancer types sequenced by the MSK-IMPACT assay was downloaded from the cbioportal website after submitting the query "STK11" in the input box. We downloaded 1661 pieces of STK11 gene mutation data and 1661 pieces of survival data. Each piece of data contained the somatic mutation status of STK11 gene, the survival time and the status of patient (decreased or censored), then we analyzed 1661 pieces of data from 1661 advanced cancer patients.

No statements of approval or informed consent were required for our study as we obtained data from an open access database.

\section{STATISTICAL METHODS}

We compared the survival curves using Kaplan-Meier method and log-rank tests. Data of overall survival (OS) was obtained from the cbioportal database directly. The overall survival was defined as the time between the day of first ICIs treatment and the date of death or last follow-up visit [2]. The prognostic significance of somatic mutation of STK11 gene after adjustment by other prognostic factors was assessed by Cox proportional hazards regression. Statistical analyses were performed using SPSS (version 17.0, SPSS Inc. Chicago, IL) and GraphPad Prism 7. Statistical significance ( $\alpha$-value) was set at 0.05 , and all $P$ values are two-sided.

\section{RESULTS}

\section{Somatic mutation analysis of STK11 gene}

We queried STK11 gene in 1661 patients, and patients with any of somatic mutation of STK11 gene would be assigned to the STK11 gene mutation group.
STK11 gene is altered in 102 of 1661 queried patients. The total somatic mutation frequency of STK11 gene among all patients was $6.14 \%$. Somatic gene mutation of STK11 was mainly distributed in patients with nonsmall cell lung cancer, which possessed more than $20 \%$ of STK11 somatic mutation.

\section{Prognostic value of somatic mutation of STK11 gene in metastatic cancer patients treated with ICIs}

We analyzed the prognostic value of somatic gene mutation of STK11 by mutation or not. KaplanMeier survival curve indicated that STK11 gene mutation was a negative prognostic factor for overall survival time. The median survival time for mutated STK11 gene and wild type STK11 gene was 7.0 months and 19.0 months, respectively, $(P<0.0001)$ (Fig. 1 A), HR increased to $1.746(95 \% \mathrm{CI}: 1.251-2.438)$.

We further analyzed the prognostic value of somatic mutation of STK11 gene in different types of tumors. As showed in figure 1, the STK11 gene mutation was significantly associated with shorter survival in NSCLC $(P=0.017)$ (Fig. 1 B). In cancer of unknown primary, the association was adjacent to statistically significance $(P=0.061)$ (Fig. $1 \mathrm{C})$, but the $\mathrm{HR}$ increased to 2.184 (95\%CI: 0.6723 -7.098). Stratified by TMB, STK11 mutation were associated with shorter survival time in both high TMB group (median OS, 47 versus 13 months, $P<0.0001$ ) and low TMB group ( median OS, 16 versus 6 months, $P<0.0001)$.

To confirm the prognostic value of somatic mutation of STK11 gene in patients treated with ICIs, we performed the Cox proportional hazards regression analysis. The results showed the somatic mutation of STK11 gene was negatively associated with overall survival, with adjustment for age, gender, drug types of ICIs, tumor types and TMB levels (Table 1).
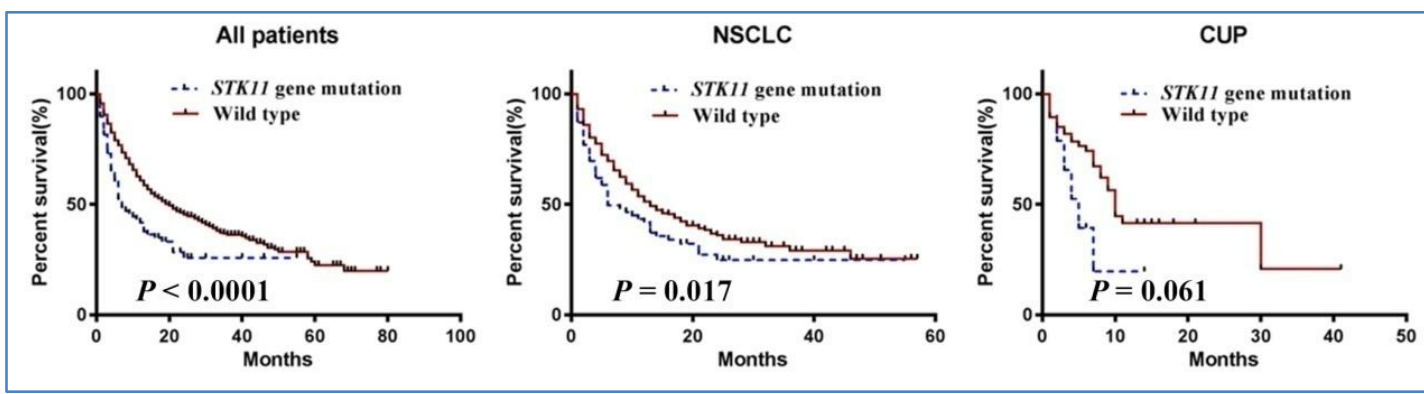

Fig-1: Kaplan-Meier survival curve showed the somatic mutation of STK11 gene was significantly associated with shorter survival in the entire cohort (A) and in patients with NSCLC (B), and adjacent to significance in patients with cancer of unknown primary(C) 
Table-1: Hazard ratios for overall survival

\begin{tabular}{|l|c|c|c|}
\hline & HR & $\mathbf{9 5 \%}$ CI & P value \\
\hline Status of STK11 gene & 1 & & 0.033 \\
\hline Wild type(reference) & 1.353 & $1.025-1.786$ & \\
\hline Mutation & & & \\
\hline & & & $<0.0001$ \\
\hline Drug type & 1 & & \\
\hline CTLA-4 (reference) & 1.405 & $1.025-1.786$ & 0.022 \\
\hline PD-1/PDL-1 & 0.782 & $0.557-1.100$ & 0.158 \\
\hline Combo & & & \\
\hline & & & 0.002 \\
\hline Tumor type & 1 & & \\
\hline Non-small lung cancer (reference) & 0.768 & $0.648-0.911$ & 0.002 \\
\hline Other types of cancer & 1.121 & $0.785-1.599$ & 0.530 \\
\hline Cancer of unkown primary & & & \\
\hline & & & $<0.0001$ \\
\hline TMB & 1 & & \\
\hline Other scores of TMB(reference) & 0.458 & $0.365-0.575$ & \\
\hline Top 20\%TMB & &
\end{tabular}

The correlation between somatic mutation status of STK11 gene and TMB score

To confirm that somatic mutation of STK11 gene may affect tumor mutation load, we performed the correlation analysis between somatic mutation status of STK11 gene and TMB score. Among all 1661 patients, there was no significant difference in TMB score between STK11 mutant group and wild-type group. Stratified by different types of cancer, the association remained no significance in none-small cell lung cancer and cancer of unknown primary, suggesting that STK11 mutation may affect the efficacy of ICIs through other mechanisms.
The correlation between somatic mutation status of STK11 gene and other genes with highest frequency

To clarify why somatic mutation of STK11 gene is associated with OS in cancer patients receiving ICIs treatment, we screened high frequency gene profiles which may correlated with STK11 gene by comutations or mutual exclusivity.

Compared with the STK11 gene unaltered group, 3 gene mutation frequencies in the STK11 gene altered group were significantly increased(Table 2), and 2 gene mutation frequencies in the STK11 gene altered group were significantly decreased(Table 2). These results suggest that STK11 mutations may affect the efficacy of ICIs by interacting with these significantly related genes.

Table-2: The top five genes with highest frequency between the STK11 gene altered group and the STK11 gene unaltered group

\begin{tabular}{|c|c|c|c|}
\hline \multirow{2}{*}{ Gene name } & \multicolumn{2}{|c|}{ Mutation frequency (\%) } & \multirow{2}{*}{ P-value } \\
\cline { 2 - 3 } & STK11 gene altered & STK11 gene unaltered & \\
\hline KEAP1 & 47.06 & 3.21 & $1.00 \mathrm{e}-36$ \\
\hline KRAS & 46.08 & 11.48 & $2.39 \mathrm{e}-14$ \\
\hline SMARCA4 & 21.57 & 7.06 & $8.25 \mathrm{e}-4$ \\
\hline TERT & 12.75 & 32.46 & $8.25 \mathrm{e}-4$ \\
\hline PTEN & 00.00 & 8.53 & $1.68 \mathrm{e}-13$ \\
\hline
\end{tabular}

\section{DISCUSSION}

Recently, immune checkpoint inhibitors (ICIs) have made remarkable progress in solid tumor therapy, especially in lung cancer and melanoma. Primary resistance is one of the main reasons why most patients do not benefit from ICIs treatment. Finding appropriate biomarkers to distinguish patients who are sensitive to ICIs from those who are not is one of the hot topics in tumor research. Recent studies have reported that high level of TMB may be related to survival benefit of ICIs treatment, and somatic mutation of STK11 gene may be one of the important markers of primary ICIs resistance. A retrospective clinical trial from Memorial sloankettering Cancer Center showed that metastatic cancer patients with high TMB score were more likely to benefit from ICIs therapy[2]. In order to clarify whether STK11 gene mutation could be used as a prognostic marker for cancer patients receiving ICIs therapy, we downloaded relevant clinical data and somatic gene mutations of STK11 from the same cohort of cancer patients receiving ICIs therapy by cBioportal database. We analyzed the relationship between somatic gene 
mutations of STK11 and overall survival. The results showed somatic gene mutations of STK11 was significantly associated with shorter overall survival in all patients and in patients with NSCLC (Figure 1), and somatic gene mutations of STK11 is an independent negative prognostic factor for cancer patients treated with ICIs (Table 1).

Serine/threonine kinase 11 (STK11), also known as liver kinase B1 ( $L K B 1)$, encoding a serine threonine kinase with an important role in the regulation of cellular metabolism, energy homeostasis and growth, is a major tumor suppressor gene[12]. The inactivated STK11 gene mutation may affect ICIs efficacy by inhibiting the expression of PD-L1, releasing large amounts of inflammatory factors and inhibiting the accumulation of tumour infiltrating lymphocytes (TILs) in tumor regions[8, 1315].STK1 1/LKB1 alterations may be a major driver of primary resistance to PD-1 blockade in LUAC [8, 14, 16].Our study showed somatic gene mutations of STK11 was a negative prognostic factor for overall survival time among all patients and in patients with NSCLC $(P<0.0001$ and $P=0.017)$ (Fig. $1 \mathrm{~A}$ and 1B). In cancer of unknown primary, the association was adjacent to statistically significance $(P=0.061)$ (Fig. 1 C), but the HR increased to 2.184 (95\%CI: 0.6723 7.098).The negative prognostic effect of STK11 gene mutation on the efficacy of ICIs was not affected by TMB levels. Even in high TMB group, STK11 mutation also was associated with shorter survival time. After adjustment for age, gender, drug types of ICIs, tumor types and TMB levels, Cox proportional hazards regression analysis showed the somatic mutation of STK11 gene was is an independent negative prognostic factor for overall survival in metastatic cancer patients treated with ICIs (Table 1).

Somatic mutation of STK11 gene has organ selectivity, which mainly occurs in non-small cell lung cancer. In our study, the total somatic mutation frequency of STK11 gene among all patients was $6.14 \%$, and more than $20 \%$ in patients with non-small cell lung cancer, which may explain why somatic mutation of STK11 gene also could be a negative prognostic factor for overall survival in patients with NSCLC.

The inactivated somatic mutation of STK11 gene may affect efficacy of ICIs by interfering with the immune microenvironment through mutation of cooccurrence or mutual exclusivity with other oncogenes and tumor suppressor genes[15]. In our analysis, 3 gene mutation frequencies in the STK11 gene altered group were significantly increased (Table 2), and 2 gene mutation frequencies were significantly decreased (Table 2). These results suggest that STK11 mutations may affect the efficacy of ICIs by interacting with these significantly related genes. STK11 may be a new target for tumor therapy $[12,17]$. The mechanism of STK11 gene mutation participating in the therapeutic effect of ICIs is not very clear, which needs further clinical and basic research.

\section{CONCLUSIONS}

Somatic gene mutation of STK11 is an independent negative prognostic factor for cancer patients treated with ICIs. The inactivated STK 11 gene mutation may affect efficacy of ICIs by interfering with the immune microenvironment through mutation of cooccurrence or mutual exclusivity with other oncogenes and tumor suppressor genes.

\section{ACKNOWLEDGEMENT}

The present study was supported by the Science and Technology Planning Project of Changzhou, Jiangsu Province (Grant no. CE20165052), the Changzhou Health Bureau Project (Grant no. ZD201616), the Jiangsu Province Health Department Project (Grant no. Z201616), the "333 Talents Training Project" of Jiangsu Province (Grant nos. 2016 III-0727 and BRA2017114), and the "Talents Training Project" for the Key Medical Innovation of Changzhou (Grant no. 2016CZLJ021).

\section{Competing interests}

The authors declare that they have no competing interests

\section{REFERENCES}

1. Erhardt, N. L., Werbel, J. D., \& Shrader, C. B. (2003). Board of director diversity and firm financial performance. Corporate governance: $A n$ international review, 11(2), 102-111.

2. Samstein, R. M., Lee, C. H., Shoushtari, A. N., Hellmann, M. D., Shen, R., Janjigian, Y. Y., ... \& Kaley, T. J. (2019). Tumor mutational load predicts survival after immunotherapy across multiple cancer types. Nature genetics, 51(2), 202-206.

3. Hellmann, M. D., Ciuleanu, T. E., Pluzanski, A., Lee, J. S., Otterson, G. A., Audigier-Valette, C., ... \& Borghaei, H. (2018). Nivolumab plus ipilimumab in lung cancer with a high tumor mutational burden. New England Journal of Medicine, 378(22), 2093-2104.

4. Yi, M., Jiao, D., Xu, H., Liu, Q., Zhao, W., Han, X., \& Wu, K. (2018). Biomarkers for predicting efficacy of PD-1/PD-L1 inhibitors. Molecular cancer, 17(1), 1-14.

5. Grizzi, G., Caccese, M., Gkountakos, A., Carbognin, L., Tortora, G., Bria, E., \& Pilotto, S. (2017). Putative predictors of efficacy for immune checkpoint inhibitors in non-small-cell lung cancer: facing the complexity of the immune system. Expert review of molecular diagnostics, 17(12), 1055-1069.

6. Zehir, A., Benayed, R., Shah, R. H., Syed, A., Middha, S., Kim, H. R., ... \& Hellmann, M. D. 
(2017). Mutational landscape of metastatic cancer revealed from prospective clinical sequencing of 10,000 patients. Nature medicine, 23(6), 703.

7. Guibert, N., Jones, G., Beeler, J. F., Plagnol, V., Morris, C., Mourlanette, J., ... \& Pradines, A. (2019). Targeted sequencing of plasma cell-free DNA to predict response to PD1 inhibitors in advanced non-small cell lung cancer. Lung Cancer, 137, 1-6.

8. Skoulidis, F., Goldberg, M. E., Greenawalt, D. M., Hellmann, M. D., Awad, M. M., Gainor, J. F., ... \& Ali, S. M. (2018). STK11/LKB1 mutations and PD-1 inhibitor resistance in KRAS-mutant lung adenocarcinoma. Cancer discovery, 8(7), 822-835.

9. Koyama, S., Akbay, E. A., Li, Y. Y., Aref, A. R., Skoulidis, F., Herter-Sprie, G. S., ... \& Diao, L. (2016). STK11/LKB1 deficiency promotes neutrophil recruitment and proinflammatory cytokine production to suppress T-cell activity in the lung tumor microenvironment. Cancer research, 76(5), 999-1008.

10. Cerami, E., Gao, J., Dogrusoz, U., Gross, B. E., Sumer, S. O., Aksoy, B. A., ... \& Antipin, Y. (2012). The cBio cancer genomics portal: an open platform for exploring multidimensional cancer genomics data.

11. Gao, J., Aksoy, B. A., Dogrusoz, U., Dresdner, G., Gross, B., Sumer, S. O., ... \& Cerami, E. (2013). Integrative analysis of complex cancer genomics and clinical profiles using the cBioPortal. Sci. Signal., 6(269), pl1-pl1.

12. Shackelford, D. B., \& Shaw, R. J. (2009). The LKB1-AMPK pathway: metabolism and growth control in tumour suppression. Nature Reviews
Cancer, 9(8), 563.

13. Schabath, M. B., Welsh, E. A., Fulp, W. J., Chen, L., Teer, J. K., Thompson, Z. J., ... \& Antonia, S. J. (2016). Differential association of STK11 and TP53 with KRAS mutation-associated gene expression, proliferation and immune surveillance in lung adenocarcinoma. Oncogene, 35(24), 3209.

14. Kadara, H., Choi, M., Zhang, J., Parra, E. R., Rodriguez-Canales, J., Gaffney, S. G., ... \& Yoo, Y. (2017). Whole-exome sequencing and immune profiling of early-stage lung adenocarcinoma with fully annotated clinical follow-up. Annals of Oncology, 28(1), 75-82.

15. Skoulidis, F., Byers, L. A., Diao, L., Papadimitrakopoulou, V. A., Tong, P., Izzo, J., ... \& Zhang, J. (2015). Co-occurring genomic alterations define major subsets of KRAS-mutant lung adenocarcinoma with distinct biology, immune profiles, and therapeutic vulnerabilities. Cancer discovery, 5(8), 860-877.

16. Rizvi, H., Sanchez-Vega, F., La, K., Chatila, W., Jonsson, P., Halpenny, D., ... \& Hollmann, T. (2018). Molecular determinants of response to anti-programmed cell death (PD)-1 and antiprogrammed death-ligand 1 (PD-L1) blockade in patients with non-small-cell lung cancer profiled with targeted next-generation sequencing. Journal of Clinical Oncology, 36(7), 633.

17. Kawashima, I., Mitsumori, T., Nozaki, Y., Yamamoto, T., Shobu-Sueki, Y., Nakajima, K., \& Kirito, K. (2015). Negative regulation of the LKB1/AMPK pathway by ERK in human acute myeloid leukemia cells. Experimental hematology, 43(7), 524-533. 